\title{
COMPUTATIONAL FLUID DYNAMICS SIMULATION OF ABRASIVE FLOW MACHINING OF BIOMATERIALS
}

\author{
${ }^{*}$ Sai Venkata Phanindra Chary Arendra, ${ }^{2}$ Pavan Durga Sai Uppuluri, and ${ }^{3}$ Varun Raju Vyasa \\ Bhattu \\ 1, 2, 3 Student, Department of Automobile Engineering, VNR Vignana Jyothi Institute of Engineering and Technology, \\ India; \\ Email: $\left\{^{* 1}\right.$ sai.arendra16298@gmail.com, ${ }^{2}$ upavanchowdary@gmail.com, ${ }^{3}$ varunraju.vb@gmail.com\}
}

\begin{abstract}
Surface finishing of the external surfaces and comfortably reachable internal surfaces to a certain specification can be attained by traditional machining processes. However, most of these processes cannot finish internal and external surfaces of intricate shapes as they are nonflexible in nature. For this type of problem Abrasive Flow Machining (AFM) can be a viable solution. The AFM process uses a self-deforming tool. In the areas where the flow is restricted the abrasion takes place removing the material and generating finer surfaces. As time passed, different kinds of AFM have been developed to maximize productivity and enhance the surface finish. Factors that influence the material removal and surface finish in the AFM process are particle size, extrusion pressure, piston velocity, and media viscosity. Carrying out experiments for all the process parameters and obtaining better process parameters of AFM, precisely, in less time is tough to achieve. Computational Fluid Dynamics (CFD) simulation was employed to determine machining parameters. In this project, the parameters obtained theoretically and from the CFD simulation are compared with the available experimental data and then the better machining parameters for the surgical implant materials ASTM F1537 wrought Co-28Cr-6Mo alloy and Ti6Al4V alloy were also determined by using CFD simulation.
\end{abstract}

Key Words - Abrasive Flow Machining, Self-deforming tool, Extrusion Pressure, Surface roughness, Computational Fluid Dynamics

\section{Introduction}

Abrasive Flow Machining (AFM) is a non-traditional machining method that is applied to polish, deburr, chamfer, produce compressive residual stresses, and remove recast layers. AFM was initially introduced and patented in 1970, by McCarty, the president of Extrude Hone Corporation.

In the AFM process, a semi-solid media containing carrier (polymer base) and abrasive particles in the desired proportion, under certain pressure is extruded across the surface that has to be finished. Whenever forced through some restrictions, the media behaves like a flexible tool. The attribute that allows the media to flow through the intricate shapes is deformation. At the surfaces to be finished by AFM, the restricted passages for the media flow are compulsory, wherein the media deports remotely like a flexible grinding stone, abrades the workpiece, and gives a good finish for the workpiece surface. Generally, a fixture is employed to create restrictions thereby directing the media in or on the workpiece to desired locations. The abrasive grains that are commonly used are made of silicon carbide. The other abrasive particles used are made of diamond particles, aluminum oxide, boron carbide. Generally, polyborosiloxane, a natural organic polymer of alkanes,

ISSN (Print): 2456-6411 | ISSN (Online): 2456-6403 alkenes, esters, and sulfoxides, and polypropylene glycol, polyethylene glycol, etc. are used as a carrier [1].

The abrasion in AFM is described as the material removal from the workpiece surface by abrasion from abrasive particles, uni-directionally, which are always of another material [2]. In general, the mechanism of material removal, which has been researched by numerous researchers, is the abrasive particle's scratching activity. The penetration of the spherical grains into the workpiece's surface occurs due to the normal force acting on the grains. The penetrated grain, as it moves horizontally i.e. along the workpiece, removal of the material takes place. The total quantity of stock material removed is the same as the complete volume of grooves generated by each grain in the extrusion media on the workpiece's surface. The quantity of stock material removed and surface roughness obtained can be evaluated if the depth and the shape of the grooves generated, and quantity of active abrasive grains are known. The following modes are involved in the mechanism of material removal and surface finish [1]:

- Elastic deformation, corresponding to rubbing

- Ploughing or plastic deformation, where most of the material is displaced without being removed

- Micro cutting, where removal of the material in the form of chips takes place

165 JREAS, Vol. 06, Issue 04, Oct 2021 
The depth of penetration, the flow of media, the magnitude of cutting forces, and workpiece properties determine the occurrence of any of these deformation modes.

In general, the AFM system consists of components like a hydraulic cylinder, hydraulic pump, pressure gauge, pistons, abrasive medium cylinder, and tooling and fixture arrangements for the workpiece. Machine, media, and tooling are the 3 crucial constituents of the AFM system if the operating and range of machining in AFM are focused.

In the AFM process, to get the better machining parameters for a specimen experimentally, numerous experiments have to be performed varying the input parameters, since different input parameters give different output results. This procedure to find better machining is difficult, time taking, and not cost-effective.

Therefore the analysis of the AFM process numerically, with the aid of Computational Fluid Dynamics (CFD) software is performed. Then by obtaining the outputs from the software and substituting them in the equations gives the results. Since the simulation is done with the aid of computers, any number of input parameters can be varied to get different outputs and thus better input parameters can be predicted.

Bio-implants can be outlined as prostheses that are utilized to normalize the physiological functions of the body. They are prepared from biosynthetic materials such as collagen, and tissue-engineered materials like artificial tissues or skin. Bioengineered devices such as artificial orthopedic implants, and cardiac pacemakers which are placed completely inside the patient's body, also come under bio-implants.

Spinal implants are the devices that are used to treat deformity of the spine, strengthen and stabilize the spine, and assist in fusion, by the surgeons, during surgery. They are also used to treat disorders like scoliosis, degenerative disc disease, kyphosis, fracture, and spondylolisthesis. Surgical grade stainless steel (generally SAE 316L grade), pure titanium (Ti) or titanium alloys, and cobaltchromium (Co-Cr) alloys are the metals used for implants. Generally, CNC machines are used for manufacturing metal implants which generally result in average surface roughness of 0.6 microns.

To increase their surface finish, the implants contain some kind of surface modification. This is because numerous studies showed favourable results of roughness clinically, in vivo, and, in vitro. Various surface modification methods are available to decrease roughness. For example, sandblasting, acid etching, anodic oxidation, and, heat treatments, and, also the amalgamation of any of these methods. The surface topography produced by these various methods differs widely.

Not only in osseointegration but surface roughness also play a vital role in the reduction of microorganism's growth. The lower the surface roughness the lesser will the accumulation of foreign contaminants and which leads to longer implant life and better user health.
The triumph of spinal implants mostly depends upon its surface characteristics like surface energy, surface chemistry, surface roughness, etc., along with the design of the implant. To attain better osseointegration, surface roughness is regarded as the primary surface characteristic of any other surface characteristics. Therefore the value of surface roughness determines the rate of osseointegration, $\mathrm{BIC}$, and thus the quality of the implant. The preferred surface roughness for spinal implants is 0.2 microns [2].

Jain VK and Adsul SG [3] concluded that the workpiece's inceptive surface roughness and hardness influence the removal of material during the AFM process. With the rise of abrasives concentration in the medium the rate of material removal rises. They also concluded that among all the process parameters investigated, the most influential parameter is the concentration of abrasives followed by the size of abrasives, and the number of cycles. They found the active grain density in the media by adding up the no of shining grains over the media surface. They also concluded that the active grain density rises with the mesh number and abrasives concentration.

P. M. Dixit, V. K. Jain, and R. K. Jain [4] made investigations into the mechanism of the AFM process and evaluated the stresses and forces developed during the process. They also proposed theoretical analysis to predict the surface roughness and material removal in the AFM.

The AFM experimental results by V. K. Gorana, G. K. Lal, and V. K. Jain [5] have showcased that the axial and radial forces, grain depth of penetration, and the density of active grains, all have a considerable effect on the material deformation.

V. K. Gorana, V. K. Jain, and G. K. Lal [6] proposed an analytical model to predict and simulate the surface finish for various machining parameters in AFM. Active grain density was found to rise with the rise in the percent of abrasive concentration and also with the rise in extrusion pressure of the medium during the AFM process. This in turn raises the reduction in $\mathrm{Ra}$ value. Another important parameter in the AFM is the initial Ra of the workpiece.

Pawan Pal, K K Jain [7] employed Computational fluid dynamics simulation to determine better machining parameters for the AFM process, accurately. He designed a 2D model, performed the flow analysis on it, and compared the results with available experimental data.

Somashekhar S. Hiremath [8] carried out the AFM on nitralloy collar sleeves and the results obtained showed a considerable reduction in surface roughness value with the increase in no of passes. It was also observed that there was only a marginal enhancement in the surface texture after a certain no of passes.

T.R. Loveless, R.E. Willams, K.P. Rajurkar [8] investigated the AFM effects on surfaces that are produced by milling, turning, wire electrical discharge machining, and grinding. The statistical analysis showed that the machining process type showed effects on material removal and surface finishing achieved. The initial condition of the surface influenced the quantity of material removed. The material removal ability of tremendously 
viscous media was observed to be about three hundred times higher than that of the lower viscous media. The chief parameters that affect the stock material removal and media velocity are the concentration of abrasives, abrasive particle size, and media viscosity.

Gudipadu Venkatesh, Apurbba K Sharma, Manjot S Cheema, and Akshay Dvivedi [1] made a study on developments in AFM Process. They also stated that the surface roughness drops with the rise in no of cycles up to a certain no of cycles and then it increases.

Rolando A. Gittens, Zvi Schwartz, Rene OlivaresNavarrete, and Barbara D. Boyan [2] Made investigations on the effect of surface roughness on the spinal implants osseointegration and concluded that at the surface roughness of 0.2 microns better results were obtained.

Rajendra Baraiya, Vivek Jain, Dheeraj Gupta [9] made studies on the existing AFM techniques and developments in them and the effect of several input parameters on the output.

M. Ravi Sankar, J. Ramkumar, and V. K. Jain [10] gave an overview of the AFM process, its types, process parameters, and their effect, major research areas in the AFM process, and recent developments in the AFM process.

The ANSYS Fluent Tutorial Guide [11] consists of several tutorials that can assist the user in the usage of ANSYS Fluent to solve various kinds of problems. In every tutorial, various options that are used for problem setup and post-processing, and viewing the results were described.

\section{Materials and Methods}

\subsection{Mathematical Modelling}

The mathematical modelling of the AFM process is done to obtain the output parameters theoretically. The workpiece taken into consideration for the present modelling is a cylindrical one as per the dimensions considered by V. K. Gorana, G. K. Lal, and V. K. Jain [6].

Following assumptions were made in theoretical modeling:

1. The abrasive particles are spherical in shape and every abrasive particle contains only one active cutting edge.

2. For the convenience of mathematical calculation, the force on each abrasive particle is assumed to be constant and is the same as the average force on all the grains.

3. The same depth of indentation is achieved by every abrasive particle due to the force applied.

4. The profile is assumed to have uniform surface roughness.

\subsection{CFD Simulation}

The mathematical representation of the flow of the media in the AFM process includes continuity, momentum, and constitutive equations. To study the flow of the media, a CFD simulation is performed. Simulation of fluid flow is included in various commercial software packages. ANSYS FLUENT was used for performing the CFD simulation in this work. The simulation was performed by utilizing a multiphase mixture model by assuming the fluid to be Newtonian and the flow to be laminar, steady-state, and incompressible. The carrier media used in for AFM process is an intense viscous one that generally is forced with the aid of a piston. Therefore the influence of the gravitational force is neglected. The flow is modelled for only one stroke. The geometry of the workpiece was generated in the design modeller of the ANSYS FLUENT according to dimensions considered by V. K. Gorana, G. K. Lal, and V. K. Jain [6] in 2D and taken as an axissymmetric model. The generated geometry and mesh are shown in the following figures. The same geometry and mesh are used for the spinal implant materials also.

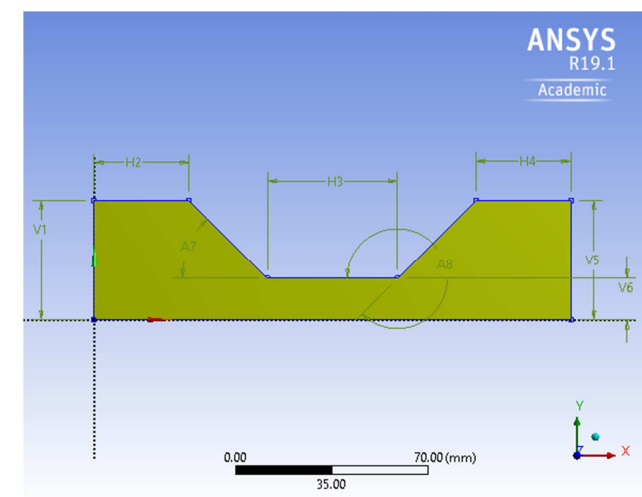

Fig. 1. Geometry of workpiece

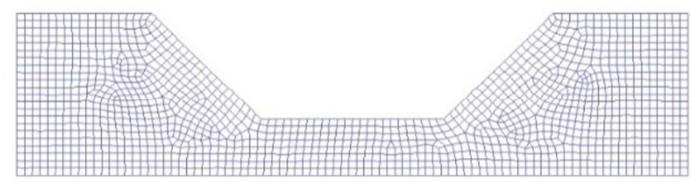

Fig. 2. Generated mesh

Dimensions, material properties of the mild steel workpiece, and machining parameters are: Radius of the workpiece $-15 \mathrm{~mm}$, Length of the workpiece $-47 \mathrm{~mm}$, Hardness of workpiece material - 2177.8 MPa, Young's modulus of workpiece material - $202086 \mathrm{MPa}$, Density of workpiece material $-0.007854 \mathrm{~g} / \mathrm{mm}^{3}$, Concentration of abrasives $-60 \%$, Radius of the media cylinder $-43.5 \mathrm{~mm}$, Stroke length - $34.5 \mathrm{~mm}$, Abrasive media polyborosiloxane (density $-1140 \mathrm{~kg} / \mathrm{m}^{3}$, viscosity -0.789 $\mathrm{kg} / \mathrm{Pa}-\mathrm{s}$ ), Abrasive - silicon carbide (density - 3170 $\mathrm{kg} / \mathrm{m}^{3}$ ), Abrasive mesh size -80 , and Extrusion pressure 4, 5, 6, 7, and $8 \mathrm{MPa}$ 


\subsection{Boundary Conditions}

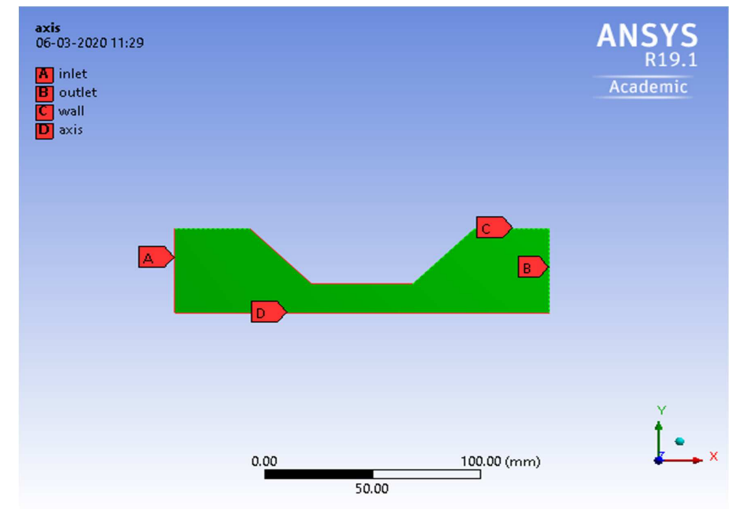

Fig. 3. Boundaries

The following boundary conditions are applied to the regions $\mathrm{A}, \mathrm{B}, \mathrm{C}$, and $\mathrm{D}$, shown in figure 3

- At inlet (A) - velocity inlet (velocity is normal to the boundary i.e. in the positive $\mathrm{X}$-axis direction and velocity in the $\mathrm{Y}$-axis direction is zero)

- No-slip at the walls (C)

- Axis of symmetric at (D)

- At outlet (B) - velocity is normal to the boundary i.e. in the positive $\mathrm{X}$-axis direction and velocity in the $\mathrm{Y}$-axis direction is zero

\subsection{CFD Simulation of Biomaterials}

CFD simulation was also performed on the materials ASTM F1537 wrought Co-28Cr-6Mo alloy and Ti6Al4V alloy, which are used for biomaterials, in a similar way as for mild steel material. From the CFD simulation performed, better machining parameters for both the material workpieces were predicted. The dimensions, material properties, and machining parameters of the biomaterial workpieces are as follows:

- For ASTM F1537 wrought Co-28Cr-6Mo alloy: Radius of the workpiece $-15 \mathrm{~mm}$, Length of the workpiece - $47 \mathrm{~mm}$, Hardness of workpiece material - 3384.45 MPa, Density of workpiece material $-0.007854 \mathrm{~g} / \mathrm{mm} 3$, Young's modulus of workpiece material - $220000 \mathrm{MPa}$, Tensile strength - $940 \mathrm{MPa}$, Concentration of abrasives $60 \%$, Radius of the media cylinder $-43.5 \mathrm{~mm}$, Stroke length - $34.5 \mathrm{~mm}$, Abrasive media polyborosiloxane (viscosity - $0.789 \mathrm{~kg} / \mathrm{Pa}-\mathrm{s}$, density - $1140 \mathrm{~kg} / \mathrm{m} 3$ ), Abrasive - silicon carbide (density - $3170 \mathrm{~kg} / \mathrm{m} 3$ ), Abrasive mesh size 400, 600, 800, and Extrusion pressure - 4, 5, 6, 7, and $8 \mathrm{MPa}$.

- For Ti6Al4V alloy: Radius of the workpiece - 15 $\mathrm{mm}$, Length of the workpiece $-47 \mathrm{~mm}$, Hardness of workpiece material $-3472.74 \mathrm{MPa}$, Density of workpiece material $-0.00443 \mathrm{~g} / \mathrm{mm} 3$, Young's modulus of workpiece material $-110000 \mathrm{MPa}$, Tensile strength - $1031 \mathrm{MPa}$, Concentration of abrasives $-60 \%$, Radius of the media cylinder $43.5 \mathrm{~mm}$, Stroke length - $34.5 \mathrm{~mm}$, Abrasive media - polyborosiloxane (viscosity - $0.789 \mathrm{~kg} / \mathrm{Pa}$ $\mathrm{s}$, density - $1140 \mathrm{~kg} / \mathrm{m} 3)$, Abrasive - silicon carbide (density - $3170 \mathrm{~kg} / \mathrm{m} 3$ ), Abrasive mesh size $-400,600,800$, and Extrusion pressure - 4, 5, 6,7 , and $8 \mathrm{MPa}$

\section{Results}

AFM is a precision finishing process and it is controlled by various process parameters such as media velocity, extrusion pressure, the media viscosity, the abrasives concentration, and the abrasive particle size. The mathematical modelling and the CFD simulation for various process parameters were analyzed to augur the results. The theoretical and CFD simulation results obtained for the materials and process parameters considered are as follow:

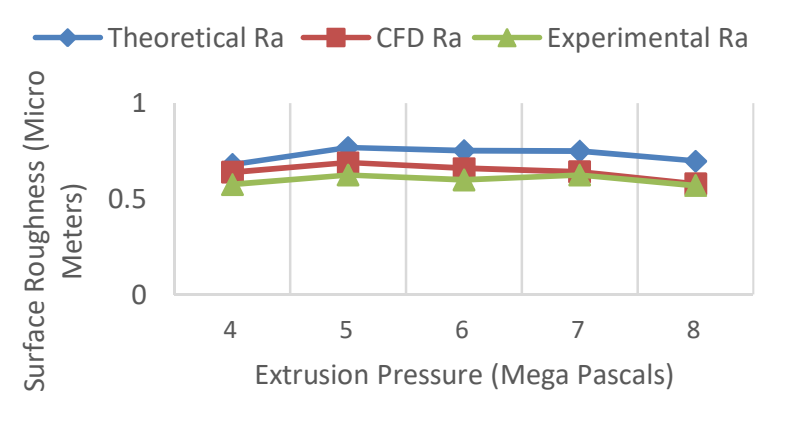

Fig. 4. Surface roughness value variation with extrusion pressure for mild steel (mesh number - 80, abrasive concentration $-60 \%$ )

The obtained results from the CFD simulation for the mild steel material are correlating with the experimental results of V. K. Gorana. G. K. Lal, and V. K. Jain [6]. From figure 4 , it was observed that the results obtained from CFD simulation lied between theoretical and experimental values. This means CFD simulation can be used to predict the AFM process results more accurately than theoretically. The results obtained from the CFD simulation for the ASTM F1537 wrought Co-28 Cr-6 Mo alloy is as follows. For better understanding, graphs were plotted to represent the results.

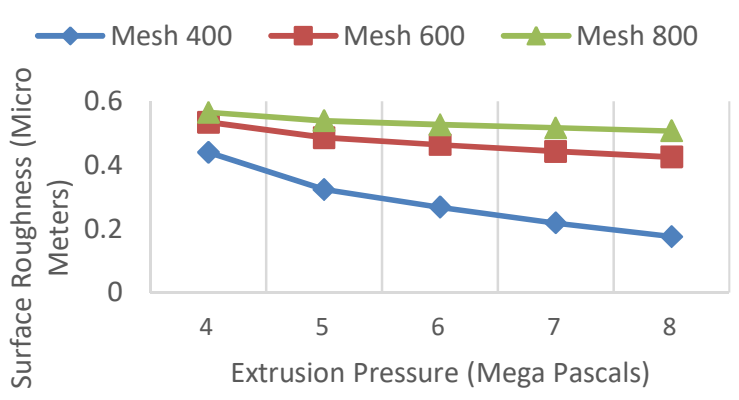

Fig. 5. Variation of surface roughness with extrusion pressure for ASTM F1537 wrought Co-28 Cr-6 Mo alloy 
for mesh numbers 400, $600 \& 800$ (abrasive concentration $-60 \%, 100$ cycles)

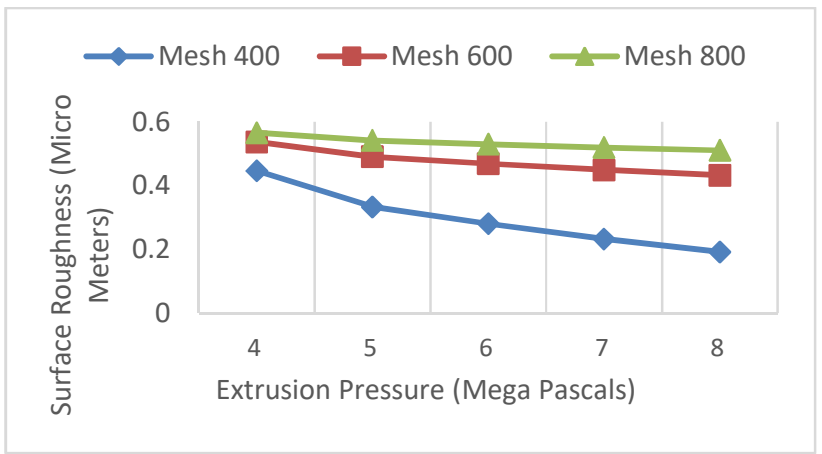

Fig. 6. Surface roughness variation with extrusion pressure for Ti6Al4V alloy for mesh numbers 400, $600 \&$ 800 (abrasive concentration $-60 \%, 100$ cycles)

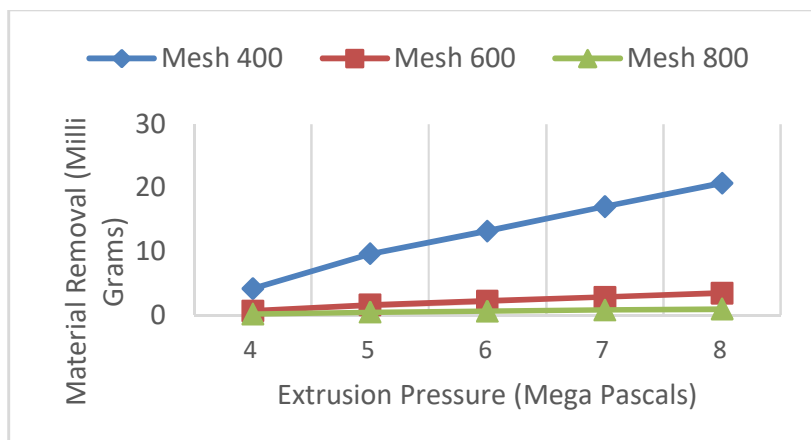

Fig. 7. Material removal variation with extrusion pressure for ASTM F1537 wrought Co-28 Cr-6 Mo alloy for mesh numbers $400,600 \& 800$ (abrasive concentration $-60 \%$, 100 cycles)

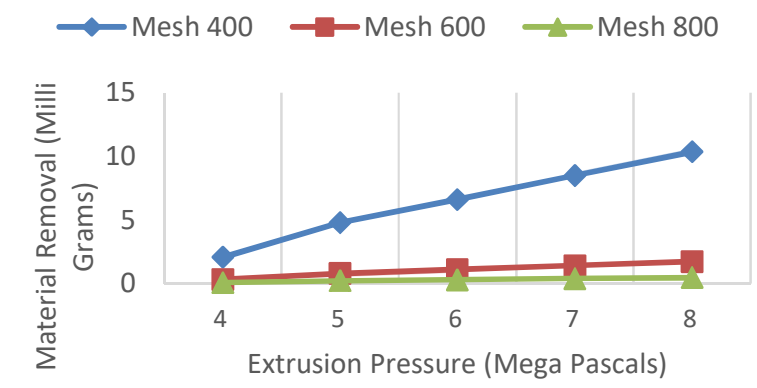

Fig. 8. Material removal variation with extrusion pressure for Ti6Al4V alloy for mesh numbers $400,600 \& 800$ (abrasive concentration $-60 \%, 100$ cycles)

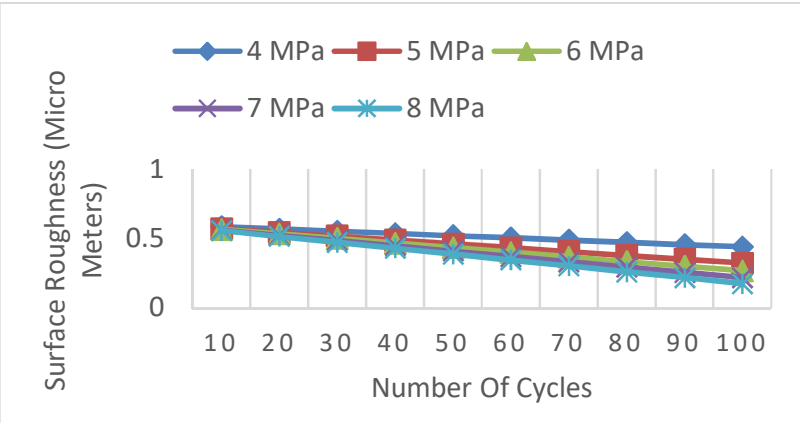

Fig. 9. Surface roughness variation with number of cycles for ASTM F1537 wrought Co-28 Cr-6 Mo alloy (abrasive concentration $-60 \%$, mesh number -400 )

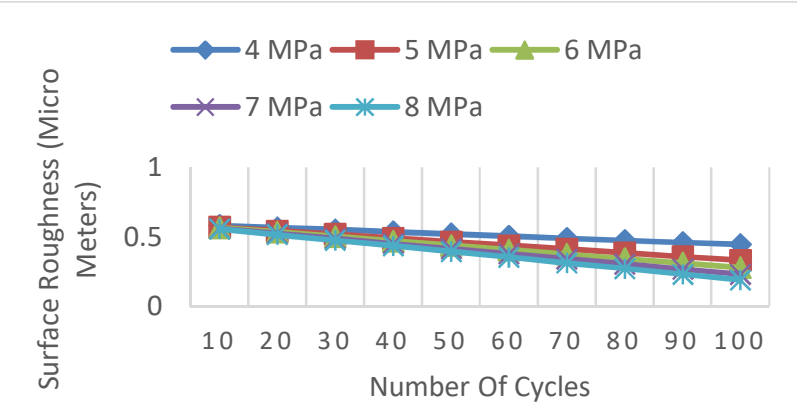

Fig. 10. Surface roughness variation with number of cycles for Ti6Al4V alloy (abrasive concentration $-60 \%$, mesh

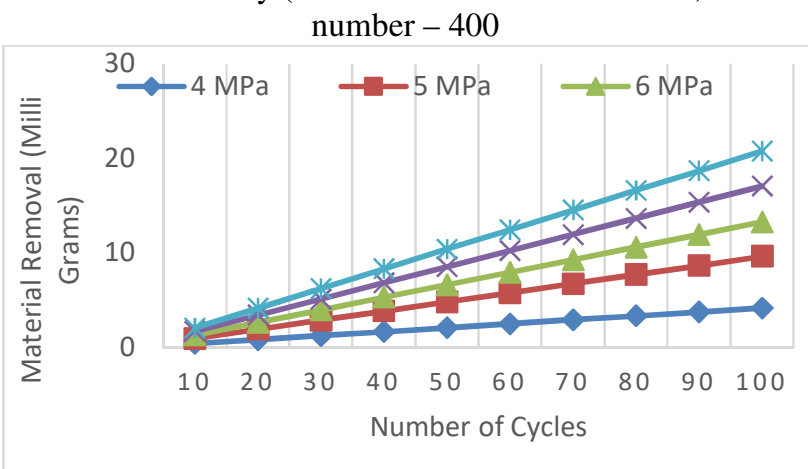

Fig. 11. Material removal variation with number of cycles for ASTM F1537 wrought Co-28 Cr-6 Mo alloy (abrasive concentration $-60 \%$, mesh number -400 )

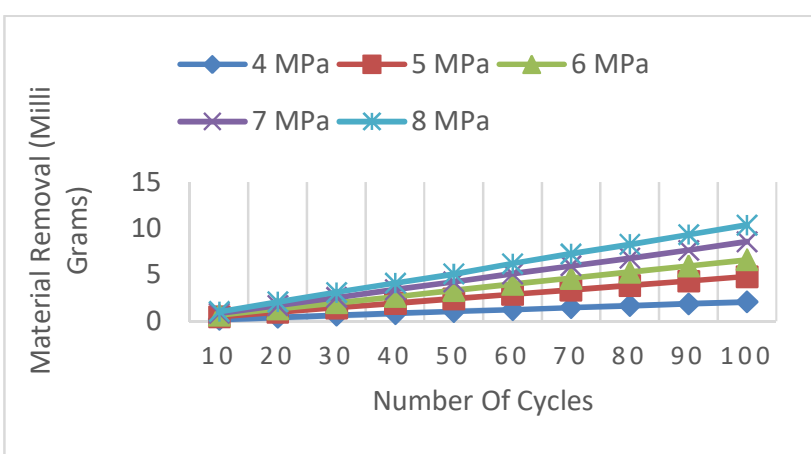

Fig. 12. Material removal variation with number of cycles 
for Ti6Al4V alloy (abrasive concentration $-60 \%$, mesh number - 400)

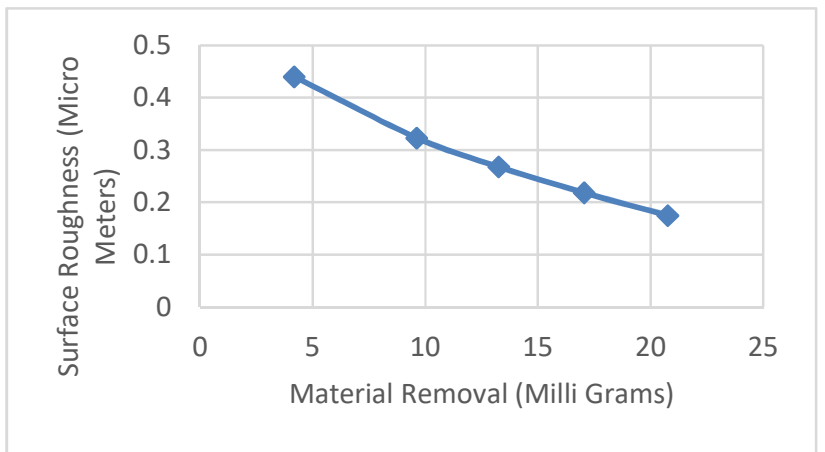

Fig. 13. Surface roughness variation with material removal for ASTM F1537 wrought Co-28 Cr-6 Mo alloy

(abrasive concentration $-60 \%$, mesh number -400 )

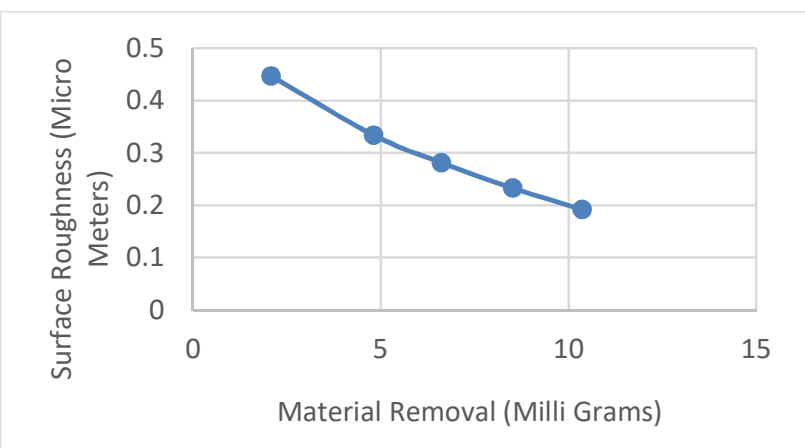

Fig. 14. Surface roughness variation with material removal for Ti6Al4V alloy (abrasive concentration $60 \%$, mesh number - 400)

Similarly, the results obtained for other process parameters were plotted. The machining parameters at which the required surface roughness is obtained in minimum time are considered to be better machining parameters

\section{Conclusions}

The following are the major conclusions drawn from CFD simulation of AFM of biomaterials:

- It was observed that the results obtained from the CFD simulation lied between theoretical and experimental values. Therefore CFD simulation can be used to predict AFM process results more accurately than theoretically.

- It was observed that the rate of material removal dropped by $83 \%$, approximately, when the mesh number increased from 400 to 600 and it dropped by $72 \%$, approximately, when the mesh number increased from 600 to 800 . Therefore, the rate of material removal drops with the rise in mesh number, can be concluded.

- It was observed that for a particular mesh number and extrusion pressure the rate of material

ISSN (Print): 2456-6411 | ISSN (Online): 2456-6403 removal increased with the no of cycles. Therefore, the rate of material removal increases with the no of cycles, can be concluded.

- It was noticed that the variation in surface roughness decreased by $60 \%$, approximately, when the mesh number increased from 400 to 600 and it decreased by $47 \%$, approximately, when the mesh number increased from 600 to 800 . Therefore, the difference in surface roughness per cycle drops with the rise in mesh number can be concluded.

- It was observed that for a particular mesh number and extrusion pressure the difference in surface roughness increased with the no of cycles. Therefore, the difference in surface roughness rises with the no of cycles, can be concluded.

- From the CFD simulation, it was found that the better machining parameters for the materials ASTM F1537 wrought Co-28Cr-6Mo alloy and Ti6Al4V alloy of the specified dimensions are 400 Mesh number, $8 \mathrm{MPa}$ extrusion pressure, and 90 cycles.

\section{Acknowledgments}

We express our thankfulness to Mr. Ch Vamshi Krishna, Assistant Professor, Department of Automobile Engineering, VNRVJIET, India, our project supervisor, for giving constant supervision, inspiring discussions, precious guidance, throughout this work. His conscientious efforts, timely help, and constructive criticism motivated us to broach this work.

We express our sincere thanks to Dr.T.Srinivasa Rao, Head of Department, Automobile Engineering, VNRVJIET, India for providing us with constant support during the project work.

We express our thankfulness to Dr. C. D. Naidu, Principal, VNRVJIET, for giving us this opportunity to carry out project work at the highly esteemed organization.

We express our thankfulness to all our well-wishers for their help.

\section{References}

[1] M. S. Cheema, G. Venkatesh, A. Dvivedi, and A. K. Sharma, "Developments in abrasive flow machining: A review on experimental investigations using abrasive flow machining variants and media," Proc. Inst. Mech. Eng. Part B J. Eng. Manuf., vol. 226, no. 12, pp. 1951-1962, 2012, doi: $10.1177 / 0954405412462000$.

[2] R. A. Gittens, R. Olivares-Navarrete, Z. Schwartz, and B. D. Boyan, "Implant osseointegration and the role of microroughness and nanostructures: Lessons for spine implants," Acta Biomater., vol. 10, no. 8, pp. 3363-3371, 2014, doi: 10.1016/j.actbio.2014.03.037.

[3] V. K. Jain and S. G. Adsul, "Experimental investigations into abrasive flow machining (AFM)," Int. J. Mach. Tools Manuf., vol. 40, no. 7, pp. 1003-1021, 2000, doi: 10.1016/S08906955(99)00114-5.

[4] R. K. Jain, V. K. Jain, and P. M. Dixit, "Modeling of material removal and surface roughness in abrasive flow machining process," Int. J. Mach. Tools Manuf., vol. 39, no. 12, pp. 19031923, 1999, doi: 10.1016/S0890-6955(99)00038-3.

JREAS, Vol. 06, Issue 04, Oct 2021 
[5] V. K. Gorana, V. K. Jain, and G. K. Lal, "Forces prediction during material deformation in abrasive flow machining," Wear, vol. 260, no. 1-2, pp. 128-139, 2006, doi: 10.1016/j.wear.2004.12.038.

[6] V. K. Gorana, V. K. Jain, and G. K. Lal, "Prediction of surface roughness during abrasive flow machining," Int. J. Adv. Manuf. Technol., vol. 31, no. 3-4, pp. 258-267, 2006, doi: 10.1007/s00170-005-0197-4.

[7] P. Pal and K. K. Jain, "Computational Simulation of Abrasive Flow Machining for Two Dimensional Models," Mater. Today Proc., vol. 5, no. 5, pp. 12969-12983, 2018, doi: 10.1016/j.matpr.2018.02.282.

[8] T. R. Loveless, R. E. Williams, and K. P. Rajurkar, "A study of the effects of abrasive-flow finishing on various machined surfaces," J. Mater. Process. Tech., vol. 47, no. 1-2, pp. 133151, 1994, doi: 10.1016/0924-0136(94)90091-4.

[9] R. Baraiya, V. Jain, and D. Gupta, "Abrasive Flow Machining: An Area Seeking for Improvement Special Issue - AETM ' 16,' IOSR J. Mech. Civ. Eng., pp. 1-9, 2016.

[10] M. R. Sankar, V. K. Jain, and J. Ramkumar, "Abrasive flow machining (AFM): An overview," Dep. Mech. Eng. Indian Inst. Technol. Kanpur, India, no. September, pp. 1-8, 2011.

[11] ANSYS Fluent Tutorial Guide 18, "ANSYS Fluent Tutorial Guide 18," ANSYS Fluent Tutor. Guid. 18, vol. 15317, no. April, pp. 724-746, 2018, doi: 10.1016/0140-3664(87)90311-2. 